\title{
Statistical fluctuations in pedestrian evacuation times and the effect of social contagion
}

\author{
Alexandre Nicolas, ${ }^{*}$ Sebastián Bouzat, ${ }^{\dagger}$ and Marcelo N. Kuperman ${ }^{\ddagger}$ \\ Consejo Nacional de Investigaciones Científicas y Técnicas, Centro Atómico Bariloche, and Instituto Balseiro, \\ R8400AGP Bariloche, Argentina
}

(Received 11 April 2016; published 23 August 2016)

\begin{abstract}
Mathematical models of pedestrian evacuation and the associated simulation software have become essential tools for the assessment of the safety of public facilities and buildings. While a variety of models is now available, their calibration and test against empirical data are generally restricted to global averaged quantities; the statistics compiled from the time series of individual escapes ("microscopic" statistics) measured in recent experiments are thus overlooked. In the same spirit, much research has primarily focused on the average global evacuation time, whereas the whole distribution of evacuation times over some set of realizations should matter. In the present paper we propose and discuss the validity of a simple relation between this distribution and the microscopic statistics, which is theoretically valid in the absence of correlations. To this purpose, we develop a minimal cellular automaton, with features that afford a semiquantitative reproduction of the experimental microscopic statistics. We then introduce a process of social contagion of impatient behavior in the model and show that the simple relation under test may dramatically fail at high contagion strengths, the latter being responsible for the emergence of strong correlations in the system. We conclude with comments on the potential practical relevance for safety science of calculations based on microscopic statistics.
\end{abstract}

DOI: 10.1103/PhysRevE.94.022313

\section{INTRODUCTION}

Large buildings or public facilities should allow a safe and quick evacuation of the attendance in the event of an emergency, such as a fire. Indeed, the uncontrolled movement of large crowds may involve excessive delays due to obstruction at narrowings and, in the most extreme cases, presents a risk of injury or even death because of pushing and trampling. To limit these risks, the design and construction of public facilities must obey strict standards, specified in building codes. In addition to design criteria that can render environments safer, a reliable prediction tool for the total evacuation time $T_{\mathrm{esc}}$, as a function, e.g., of the number $N$ of attendants, would be extremely valuable. To this end, a better understanding of the dynamical processes governing crowd motion might be crucial.

In spite of being the primary concern of much research, the mean evacuation time $\bar{T}_{\text {esc }}(N)$ does not convey enough information to assess the safety of a facility: The fact that $\bar{T}_{\text {esc }}(N)$ lies in safe bounds does not tell us how often the evacuation will be excessively long. Fluctuations are indeed expected to be large in such complex finite-size systems. Therefore, one should consider the whole distribution of total evacuation times [1] over some (uncontrolled) space of realizations, i.e., for different compositions of the attendance, states of mind, times of the day, etc.

An evacuation may be delayed by various possible factors, but a bottleneck that has turned fatal many times is the congested passage through an (insufficiently wide) exit door. Amidst many other similar tragedies, severe congestion at an exit or a narrowing was reported in the 1863 Church of the Company of Jesus fire catastrophe in Santiago (Chile) [2],

\footnotetext{
*alexandre.nicolas@polytechnique.edu

†bouzat@cab.cnea.gov.ar

${ }^{\ddagger}$ kuperman@cab.cnea.gov.ar
}

causing more than 2000 fatalities; in the 1903 Iroquois Theatre fire disaster in Chicago, with a death toll above 600 [3] (for the full story, see [4]); in the largely documented 1989 crowd disaster at the Hillsborough stadium (England) [5,6] (in this case, congestion actually occurred at the entrance, under the pressure of the incoming crowd of supporters); in the 2010 crowd disaster during the Love Parade in Duisburg (Germany) [7], where the same tunnel was used as both the entrance and the exit from the premises; in the 2004 fire disaster in the nightclub República de Cromañón in Buenos Aires [8]; and in the recent fire disaster in the Colectiv nightclub in Bucharest in October 2015 [9], where clear problems with the emergency exits were reported.

Recently, quantitative analogies have been brought to light by Zuriguel and co-workers between the flow of grains through a small orifice and pedestrian evacuation through a narrow door, at least in the controlled conditions in which the experiments were conducted [10,11], thus suggesting that the process is dominated by basic physical mechanisms. The escape dynamics were probed microscopically (i.e., at the level of the individual); in particular, statistically, the distribution of time lapses $\Delta t$ between successive egresses was shown to be well described by a power law at long jam durations $\Delta t$, regardless of the behavior prescribed to the participants. If the bulk evacuation can indeed be robustly characterized microscopically, the following approach seems very promising: Compute the statistics of time lapses associated with a given exit geometry (microscopic statistics), using existing and future recordings of real emergency evacuations (or evacuation drills in conditions as realistic as ethically possible), and infer the sought-after distribution of $T_{\text {esc }}(N)$ (macroscopic distribution) from the microscopic one, as a sum of random time lapses.

Here we wonder about the validity and relevance of such a tempting connection between the microscopic statistics and the macroscopic distribution, henceforth called the micro-macro relation for brevity. To explore this problem and illustrate our findings, we have developed a highly economical cellular 
automaton model that is loosely inspired by the granular analogy but also has a behavioral component [12-15] and reproduces semiquantitatively the experimental data of [11]. In particular, we show that the micro-macro relation may break down, notably owing to the empirically established possibility of psychosocial contagion (or behavioral imitation, i.e., an enhanced tendency of neighbors of aggressive people to behave competitively).

In Sec. II we clarify the theoretical underpinning of the micro-macro connection and expose possible causes leading to its violation. Section III is dedicated to the presentation of the model, which is then tested against experimental data in Sec. IV in the absence of social contagion. In Sec. V we implement the latter effect in the model in a simple way and study its consequences. We conclude by critically commenting on the potential interest for safety science of the micro-macro relation, beyond our specific implementation.

\section{THEORETICAL EXPOSURE OF THE PROBLEM}

Suppose that a given facility needs to be evacuated. Schematically, the individual escape towards a safe point may be decomposed into the following phases: (a) a delay before reacting to the emergency (for various possible reasons), known as premovement time, (b) a phase of relatively unconstrained motion, alone or in group, known as travel time, and (c) possible delays due to obstruction at narrowings and exits.

Here we exclusively focus on the last point. ${ }^{1}$ To this day, the question of whether this is actually the bottleneck in tragic evacuations remains controversial. If an emergency arises, precious time is often lost in the premovement phase, which is believed to take of the order of one minute [17], thus sometimes longer than the subsequent evacuation. Moreover, a significant portion of the social psychology literature of past decades questions the prevalence of competitive moves, that is, selfish rushes towards the exit causing clogs [18-22], and hints at pieces of evidence of conserved social norms and cohesive behaviors, such as the will to assist fallen people, in emergencies. Yet reports on crowd tragedies (see references above) make the occurrence of selfish rushes in some situations unquestionable. In fact, even if the individual pedestrians are intrinsically willing to cooperate, we will see with our simple model that a global competitive response can emerge and propagate in some cases.

Recently, the escape dynamics through a narrow $(\approx 70-\mathrm{cm}-$ wide) door were studied experimentally in controlled evacuation drills [11,23]. Three distinct degrees of competitiveness were successively prescribed to the participants, from the prohibition of any contact in the most cooperative settings to the permission of moderately soft pushes to elbow one's way, in the most competitive settings. In each case, for a fine characterization of the dynamics, the distribution $p(\Delta t)$ of time intervals $\Delta t$ between successive escapes was computed, with seemingly robust bulk statistics; similarly to the case of granular hopper flows, the distributions were found to be well

\footnotetext{
${ }^{1}$ As a first step, the delays due to these distinct phases can be summed additively, under the assumption of independence [16].
}

described by power laws at large $\Delta t$, viz.,

$$
p(\Delta t) \propto \Delta t^{-\alpha}
$$

where the exponent $\alpha$ decreases with increasing crowd competitiveness. Thus, long clogging events were more likely in the more competitive crowds, which resulted in longer evacuation times. Note that only values of $\alpha$ larger than 3 were measured; this implies that $\Delta t$ has a well-defined mean value and standard deviation. From this microscopic characterization of relatively competitive egresses, one may aspire to derive the practically relevant distribution $P_{N}\left(T_{\text {esc }}\right)$ of global evacuation times of $N$ evacuees over an uncontrolled space of evacuation realizations, where $T_{\mathrm{esc}}(N)=\sum_{i=1}^{N} \Delta t_{i}$ and $\Delta t_{i}$ is the time lapse between the $(i-1)$ th and $i$ th escape (for convenience, the zeroth escape is defined as the start of the evacuation). To do so, one can think of $T_{\text {esc }}$ as a sum of $N$ independent random time-lapse variables $\Delta t$ and write

$$
P_{N}\left(T_{\mathrm{esc}}\right)=p^{* N}\left(T_{\mathrm{esc}}\right)
$$

where the superscript $* N$ should be understood as a convolution product. Regardless of the nature of the distribution $p(\Delta t)$, Eq. (2) yields a direct connection between the microscopic statistics and the global distribution. In particular, if the mean value $\overline{\Delta t}$ and the standard deviation $\sigma_{t}$ of $p(\Delta t)$ are well defined, the central limit theorem implies that, in the limit of large attendance $N \gg 1$,

$$
P_{N}\left(T_{\mathrm{esc}}\right) \sim \mathcal{N}\left(N \overline{\Delta t}, \sqrt{N} \sigma_{t}\right),
$$

where $\mathcal{N}(m, \sigma)$ denotes the normal law of mean $m$ and standard deviation $\sigma .^{2}$

Theoretically, it is well known that the micro-macro connection of Eq. (2) fails if the $\Delta t_{i}$ display strong correlations, instead of being independent. Yet, for practical purpose, it is tempting to discard this mathematical caveat. Indeed, it is virtually impossible to collect sufficient data on $P_{N}$, whereas the microscopic statistics $p$ could readily be compiled from recordings of real emergency evacuations in the studied geometry (or, all ethical issues left aside, competitive evacuation drills). In how far is this neglect justified in practice?

Here we claim that, beyond the variations in the crowd composition and the correlations in pressure in the crowd, a major limitation to the micro-macro relation originates in the propagation of noncooperative alarmed behaviors. ${ }^{3}$ Although this process of behavioral contagion, whether deliberate or not, may be less widespread than traditionally thought (or even perhaps reported [18]), it did occur in major crowd disasters and very generally aggravated the situation. Therefore, it should be heeded in the extreme conditions where safety is most imperiled. A recent example that demonstrates this effect is a video showing the beginning of a crowd movement at a gathering following the 13 November 2015 terrorist attacks in Paris [24]; another arresting example is the following statement by Marshall,

\footnotetext{
${ }^{2}$ Some details about the limit distribution expected in the hypothetical case of a power law with exponent $\alpha \leqslant 3$ are provided in Appendix A.

${ }^{3}$ This contagion process is often referred to as panic spreading, but the denomination may be improper [20].
} 
based on his combat experience during World War II: "Every large panic starts with some very minor event.... Troops will always run if they see others running and do not understand why" [25]. Since competitive rushes towards the exit have an impact on the microscopic distribution $p$, contagion induces correlations that may strongly affect the global distribution $P_{N}$.

Let us illustrate this point with an extreme example. Suppose that the crowd is extremely susceptible to fear; for instance, consider the two foregoing examples of suggestible crowds. In the (unlikely) event of someone actually displaying signs of alarm, fear will quickly pervade the whole crowd. As a result, the evacuation will be either slow, if someone actually gets panicky, or fast, in the opposite case. Accordingly, the distribution of global evacuation times cannot be inferred from the sole microscopic distribution $p(\Delta t)$, because the latter, as an average, mingles escape data for different crowd states. In particular, the micro-macro relation would predict far rarer sluggish evacuations than actually occur.

\section{PRESENTATION OF THE CONTAGION-FREE MODEL}

To test these ideas in a more concrete framework, we wish to develop a minimalistic model for pedestrian evacuation dynamics, focusing on congestion effects [point (c) above]. The model should reproduce the following microscopic statistical features suggested by experiments [10], without obfuscating the picture with complex modeling details: (i) a broader-thanexponential, power-law-like tail in the distribution $p(\Delta t)$ for narrow doors, as in Eq. (8), with exponents comparable to empirical values, and (ii) an exponential-like distribution of burst sizes, where a burst is defined as a series of uninterrupted escapes.

Inspired by previous work [14], we develop a latticebased cellular automaton. Importantly, its key ingredients can loosely be interpreted in the context of granular flows, given the aforementioned analogies [10] (also see [26]), but we account for the fact that unlike fluid particles, pedestrians are responsive to the environment and may take decisions and move accordingly. The semiquantitative agreement attained by our model with respect to the specific statistical features (i) and (ii) is, as far as we know, unprecedented in cellular automata (see Appendix $\mathrm{C}$ for remarks pertaining to the observation of power laws, notably in previous works).

Briefly, agents (representing pedestrians) will be positioned on the cells of the lattice grid, with at most one agent per site, due to steric constraints. At each time step, agents target, and may move to, one of the adjacent sites. The chosen direction is controlled by a static floor field that directs them towards the exit [27]. We insist that the model is deliberately minimalistic. Among other simplifications, we make no attempt to describe the architecture of a real facility or to account for the existence of social bonds in the evacuating crowd, even though they may be important in practice [19,28].

We opt for a simple geometry, namely, a square of side $L$, with a single door of width $L_{d}$ in the middle of one of the walls. Space is divided into square cells, of unit length.

At each time step, each agent targets one of the (at most) four adjacent sites, the so-called von Neumann neighborhood, or chooses to stay at its current location. The probability of selection of a site depends on its attractiveness, quantified by the absolute static floor field

$$
A_{s}(x, y)=\bar{d}-\underbrace{\sqrt{\left(x-x_{T}\right)^{2}+\left(y-y_{T}\right)^{2}}}_{\text {proximity to target }},
$$

where $\left(x_{T}, y_{T}\right)=\left(\frac{L}{2},-L_{d}\right)$ are the coordinates of the targeted "safe point" behind the door and $\bar{d}$ is a large value that maintains the attractiveness positive in all cases. Conceptually, $A_{s}(x, y)$ can be regarded as the negative of a potential energy in a granular system. The floor field could naturally be refined by describing, e.g., wall effects, but our simple choice turned out to be satisfactory for the present study.

Furthermore, occupied sites should always be less attractive than free ones. Thus the static attractiveness is complemented with a dynamic part: Should a site be currently occupied by another pedestrian, its attractiveness will be penalized by a large constant value $H_{0}=-10$, viz., $A=A_{s}-H_{0}$, so that free sites $\left(A=A_{s}\right)$ will virtually always be preferred to occupied ones.

A priori, pedestrians should always try to move to the adjacent free site of highest attractiveness. However, this deterministic local rule traps the system in metastable configurations, with unrealistic density profiles in front of the exit. Accordingly, some stochasticity is introduced, in the form of a small amount $\eta$ of noise (which would be a measure of the amplitude of the vibrations in a granular system). A pedestrian, on site $\mu$, selects a nearby site $v$ in her vicinity (including the current site), with a probability

$$
p_{\mu \rightarrow \nu} \equiv \frac{e^{\left(A_{v}-A_{\mu}\right) / \eta}}{\sum_{\sigma \in \Lambda_{\mu} \cup\{\mu\}} e^{\left(A_{\sigma}-A_{\mu}\right) / \eta}},
$$

where the denominator is a normalization factor and $\Lambda_{\mu}$ refers to the set of sites adjacent to $\mu$. A vanishing noise intensity $\eta$ yields the same issues as the deterministic algorithm, while high $\eta$ values produce very loose pedestrian configurations; the selected intensity $\eta=1$ offers a good compromise between these extreme cases.

Once the desired sites are selected, distinct pedestrians may aim for the same site. Because this site cannot accommodate more than one agent, this generates a conflict and the physical contacts that it involves ("friction" in the terminology of [29]) hamper motion. For simplicity, we consider a limit of strong friction, in which competitive conflicts are always sterile: Nobody then moves forward.

Our simulations have shown that the foregoing rules do not lead to a power-law-tailed distribution $p(\Delta t)$, condition (i) above. This aspect turned out to be difficult to reproduce with a cellular automaton and convinced us to examine its origin in granular flows more carefully, for these systems are better understood and display similar microscopic statistics. In granular hopper flows, clogging occurs because of the formation of pressure-bearing structures such as arches [30,31]. The large time lapses $\Delta t$ between successive escapes, forming the tail of $p(\Delta t)$, are dominated by the time that vibrations take to break these arches. Lozano et al. elucidated that the extent of time an arch resists vibrations on average is controlled by its weakest point, i.e., the grain that forms the largest angle with its two neighbors in the arch [32]. Geometry, more specifically, the variable weaknesses of the arches depending on their geometry, thus plays a central role. This calls into question 
the relevance of a lattice-based model such as ours. However, we succeeded in taking into account these aspects, without introducing computationally costly geometric considerations.

We started by taking up the original distinction proposed in [14] between cooperative (patient) and competitive (impatient or selfish) pedestrians: Here, at each time step, either of the behaviors is chosen probabilistically, on the basis of one's propensity to cooperate $\left.\left.\Pi_{i}(t) \in\right] 0,1\right]$; one will behave cooperatively with probability $\Pi_{i}(t)$ and competitively with probability $1-\Pi_{i}(t)$, where $\Pi_{i}(t)$ is fixed to its intrinsic value $\Pi_{i}^{(\text {intr) }}$ in the absence of interpedestrian contagion. In effect, the two possible behaviors or strategies differ in the tolerance for not moving. Owing to their drive, competitive pedestrians find the option to stay at their current position $\left(x_{i}, y_{i}\right)$ less attractive than cooperators, the difference being set by $\Pi_{i}(t)$ :

$$
\begin{gathered}
A\left(x_{i}, y_{i}\right) \stackrel{\text { impatient }}{\longrightarrow} A\left(x_{i}, y_{i}\right)+k \ln \Pi_{i}(t), \\
A\left(x_{i}, y_{i}\right) \stackrel{\text { patient }}{\longrightarrow} A\left(x_{i}, y_{i}\right) .
\end{gathered}
$$

The constant $k$ is set to 0.5 to get results quantitatively comparable to the experimental measurements of [11]. Thus, competitive agents will be more prone to push for one of the neighboring sites than patient ones. Using Eq. (5), one can readily see that, in a given environment of cooperative and competitive agents, an impatient individual will be more likely to opt for a forward move and therefore will move faster on average, compared to their patient counterparts.

At the constriction close to the exit, an agent will only be able to move forward if, at this time step, the neighbors accept not to attempt a move to the desired site, which depends on their propensities $\Pi_{i}(t)$ via Eqs. (6) and (7). In a granular system, this would tentatively correspond to a situation in which neighboring grains in the arch move slightly backward due to the vibration, thus leaving free space to their neighbor. Finally, to account for the heterogeneous resistances of the arches, the intrinsic propensities $\Pi_{i}^{\text {(intr) }}$ are randomly (Gaussian) distributed [remember that, without contagion, $\left.\Pi_{i}(t)=\Pi_{i}^{(\mathrm{intr})}\right]$. This disorder is critical with respect to law (i).

To sum up, at each time step, (1) all pedestrians start by selecting a target site, (2a) if the target site is occupied, the pedestrian just waits, ( $2 b$ ) otherwise, the pedestrian moves to it, unless other agents are competing for it (in which case no one moves). (3) Following this first round of motion, some sites have been freshly vacated, which may allow waiting pedestrians to move to their target site. Steps (2) are thus iterated until all possibilities of motion have been exhausted.

The iterative rule (3) allows the formation of files of moving pedestrians, without voids, and avoids an artificial pulsating dynamics. Note that agents cannot move more than once during a time step (tentatively corresponding to a fraction of a second in reality).

This completes the description of the contagion-free model, that is, the model featuring fixed propensities to cooperate $\Pi_{i}(t)=\Pi_{i}^{\text {(intr) }}$ for all agents $i$. Before introducing contagion, we dedicate the next section to the study of the fixed-behavior model, which reflects the situation expected in controlled evacuations with prescribed behaviors [11].

\section{MAIN RESULTS FOR THE CONTAGION-FREE MODEL}

Though the foregoing rules seem rather sensible, it is not granted that they can reproduce the microscopic statistical laws (i) and (ii). Here we compare the numerical simulations of the contagion-free model with experimental data on pedestrian and sheep passages through a narrow door [10,11,23,33].

In Ref. [11], three levels of pedestrian cooperativeness were tested: very competitive, moderately competitive, and cooperative. We arbitrarily define the corresponding distributions of propensities $\Pi^{\text {(intr) }}$ as Gaussian distributions of standard deviation 0.2 , peaked at $\Pi_{0}=0,0.4$, and 0.8 , respectively, and truncated to the interval $] 0,1[$, but the results are qualitatively robust to variations of these specific values.

Initially, the agents are distributed randomly in the room, with a uniform distribution conditioned upon the volume exclusion constraint, i.e., having no more than one agent per cell. The initial positions are independent of the agents' intrinsic probabilities and only affect the travel time towards the door [point (b) at the beginning of Sec. II]. In our simulations, this process marks the beginning of the evacuation, but takes a negligible time compared to the clogging-induced delays [point (c)], provided that the crowd is large enough (typically, $L \geqslant 20$ for a density $\rho=0.6$ ). The evacuation ends when the last individual has left the room.

The simulations show close agreement with experimental data, reflected in the following aspects. During the simulated evacuation, the crowd adopts a semielliptic configuration in front of the exit, in broad agreement with experimental observations. As expected, the mean global evacuation time increases monotonically with the number $N$ of agents.

As in [11], a "faster-is-slower" effect [34,35] is observed, insofar as the evacuation takes longer for increasing competitiveness of the agents, i.e., going from a cooperative to a moderately competitive and then a strongly competitive crowd, although a competitive agent would move faster on average than a patient one placed in the same given environment (see Sec. III). From now on, the initial pedestrian density will always be set to $\rho=0.6$.

For a more thorough analysis, we compute the microscopic statistics of the evacuation, with the help of the PYTHON routine implemented by Alstott et al. [36] on the basis of the powerlaw analysis methods collated by Clauset et al. [37]. Starting with point (i) above, Figs. 1 and 2 present the complementary cumulated distribution (or survival function) of time lapses $\Delta t$, in various conditions. Overall, the graphs look similar to the experimental ones [11]. For the narrowest door, their tails are well fitted by power laws

$$
p(\Delta t) \sim \Delta t^{-\alpha}, \quad \alpha>3
$$

Indeed, for $L_{d}=1$, the goodness of the power-law fit vs the exponential one is always positive, meaning that the power law provides a better fit, with $p \leqslant 0.05$ (for about $10^{6}$ sample points); for strongly competitive crowds, $p$ reaches truly vanishing values, which makes extremely unlikely the possibility that the success of the power-law fit over the exponential one is due to chance. These heavy tails are largely due to the disorder in the propensities $\Pi_{i}^{\text {(intr) }}$ : If the distributions of $\Pi_{i}^{(\mathrm{intr})}$ are replaced by a Dirac peak at their mean value, the power-law fit becomes much poorer (see 


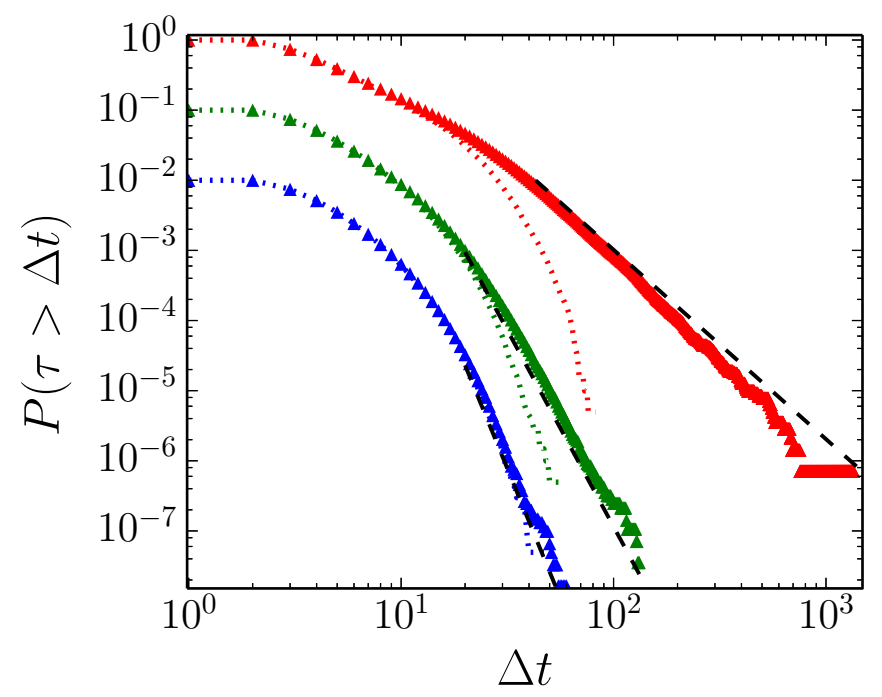

FIG. 1. Survival functions $P(\tau>\Delta t)$ for $L_{d}=1$ and different cooperativeness levels: strongly competitive (red), moderately competitive (green), and cooperative (blue), from top to bottom. The curves have been shifted vertically to improve the visibility. The dashed black lines are power-law fits, with the exponents indicated in Table I. The dotted lines are the survival functions obtained by replacing the distribution of propensities with Dirac functions peaked at their mean values.

Fig. 1). Appendix B presents an analytical endeavor towards an approximate derivation of the microscopic distribution $p(\Delta t)$ for the strongly competitive crowd, which predicts a power law with exponent $\alpha=4$ (to be compared to the numerical value $\alpha=3.7$ ).

Large time lapses $\Delta t$ become more frequent for more competitive crowds, which is reflected by a lower exponent $\alpha$, consistently with the experimental observations. The model parameters were chosen in such a way that the values of the exponents $\alpha$ tend to match those of Ref. [11] for a 70-cm-wide

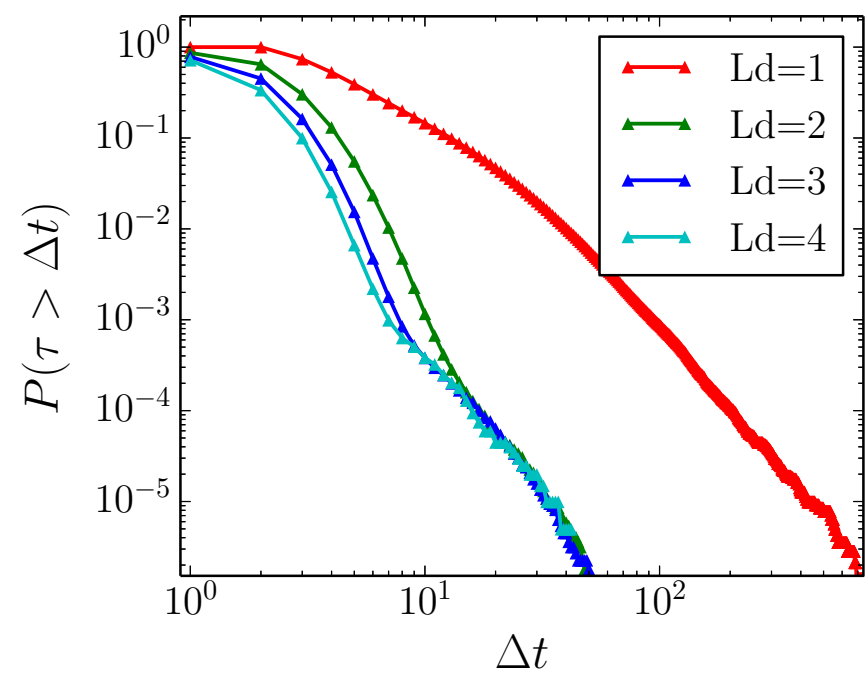

FIG. 2. Survival functions $P(\tau>\Delta t)$ for different door widths $L_{d}=1,2,3,4$, from top to bottom, with a strongly competitive crowd.
TABLE I. Values of the fitted exponents $\alpha$. The values followed by (?) are strongly dependent on the portion of the curve that is fitted and is therefore uncertain.

\begin{tabular}{lccc}
\hline \hline Behavior & $L_{d}=1$ & $L_{d}=2$ & Ref. [11] \\
\hline Strongly competitive & 3.7 & 4.3 & 4.2 \\
Moderately competitive & 6.6 & $3.8(?)$ & 5.5 \\
Cooperative & 8.4 & $5.6(?)$ & 6.8 \\
\hline \hline
\end{tabular}

door, which we here consider to be between $L_{d}=1$ and $L_{d}=2$. The (approximate) fitted values of the exponent are presented in Table I. In terms of dimensional values, assuming a time step $\delta t=0.27 \mathrm{~s}$ for cells of size $0.4 \times 0.4 \mathrm{~m}^{2}$ [38], the average time lapse $\langle\Delta t\rangle$ is comparable to the experimental value [11] for $L_{d}=2$ and overestimated by a factor 3 for $L_{d}=1$; this overestimation is not very surprising, in light of the limit of strong (infinite) friction considered in the model.

Figure 2 shows the influence of the door width. Widening the door reduces the probability of long clogs, in agreement with simulations and experiments on sheep [33], as well as with the intuition. In addition, for door widths $L_{d} \geqslant 2$, the quality of the fit of the tail with a single power law decreases. Moreover, while a dramatic change is observed as $L_{d}$ increases from 1 to 2 , variations in the distribution $p(\Delta t)$, for $\Delta t \geqslant 1$, are much more tenuous for $L_{d} \geqslant 2$. This does not imply that the outflow rate is then independent of the door width: Simultaneous escapes $\Delta t=0$ will play a more and more important role as $L_{d}$ increases. Incidentally, note that an apparent insensitivity to large door widths was also reported in [39].

The implemented dynamical rules are strictly local, so we expect the escape dynamics at the door to be mostly insensitive to finite-size effects. Indeed, for system sizes $L \geqslant 20$ (with initial density $\rho=0.6$ ), the collected microscopic statistics are virtually independent of $L$. For $L<20$, variations are perceptible, because the initial phase in which agents run towards the (still uncongested) door is no longer negligible.

The large fluctuations in the time lapses $\Delta t$ are suggestive of intermittent dynamics, in which bursts of escapes, defined as successive egresses separated by $\Delta t \leqslant 1$, alternate with long waiting times. In some experimental settings [10], these bursts of escapes were found to be distributed exponentially; see law (ii). In our cellular automaton, bursts of escapes (comprising more than one egress) are observed only for $L_{d}>1$ (the escape at $L_{d}=1$ is so sluggish that successive escapes are highly improbable); for $L_{d}=2$, for instance, Fig. 3 demonstrates that the bursts of escapes do indeed follow an exponential law. Note that this law is more readily obeyed in cellular automata than law (i), though not systematically [40].

Micro-macro relation. Since the time lapses $\Delta t$ fluctuate, the global evacuation time, which is a sum of time lapses, will also vary between realizations. These variations between realizations materialize in the distribution $P_{N}\left(T_{\text {esc }}\right)$ of global evacuation times of $N \gg 1$ agents, an example of which is shown in Fig. 4 for a competitive crowd evacuating through a narrow door. To test whether the statistical fluctuations can be deduced from $p(\Delta t)$ using the micro-macro relation, we compare the actual histogram of durations $T_{\text {esc }}$ with the Gaussian distribution predicted on the basis of Eq. (3) and 


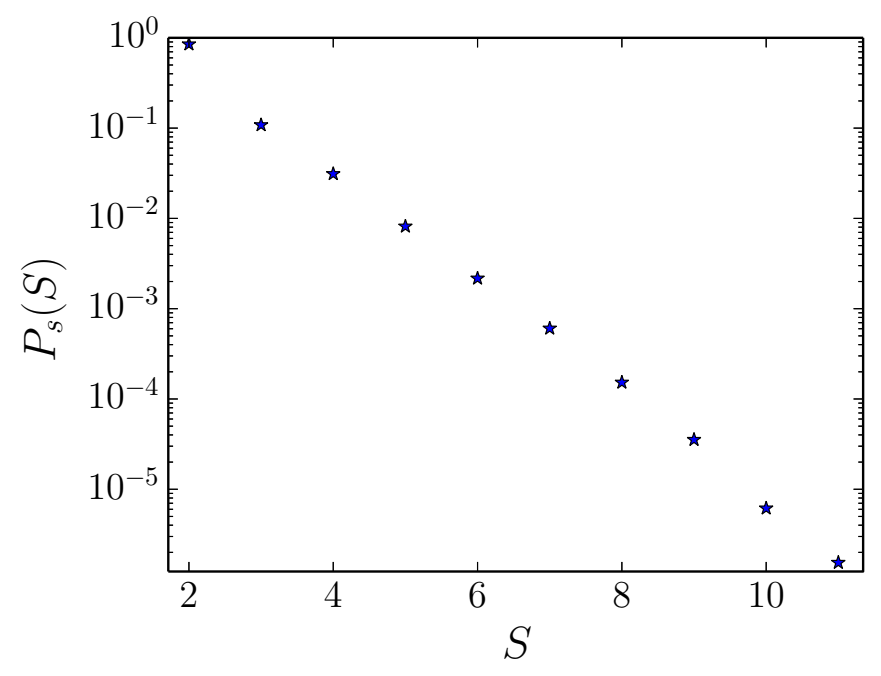

FIG. 3. Distribution of burst sizes for a door width $L_{d}=2$ and a strongly competitive crowd.

$p(\Delta t)$. The excellent agreement that is obtained validates the micro-macro relation, as expected in a situation without long correlations in the successive $\Delta t$. On a side note, let us illustrate the importance of heeding the fluctuations in the distribution, and not just the average. Suppose that safety standards were set by considering only the mean evacuation time; as a precaution, this mean value could be inflated by, say, $10 \%$ to set the norm. Still, in the particular example of Fig. 4, actual evacuations would take longer than the norm in about $8 \%$ of the realizations, even though the global evacuation time is a statistical average over $\rho L^{2} \approx 400$ evacuees.

(As an aside, we mention that for wider doors $L_{d} \geqslant 3$ we observed some anticorrelations between successive time lapses $\Delta t$, resulting in a slightly narrower global distribution of $T_{\text {esc }}$ than predicted by the micro-macro relation. This may

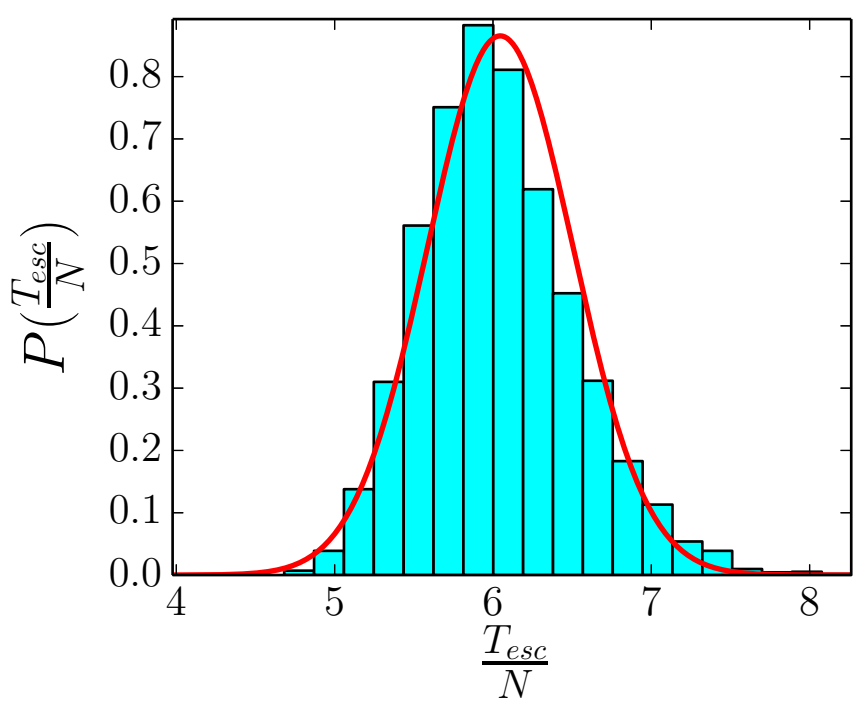

FIG. 4. Distribution of global evacuation times in the absence of contagion, for a strongly competitive crowd, $L_{d}=1$, and $L=25$. The solid red curve is the Gaussian distribution predicted on the basis of Eq. (3). be in line with experiments on pedestrian flows through bottlenecks under normal (cooperative) conditions, in which anticorrelations were also reported and ascribed to a zipper effect [41].)

\section{IMPACT OF CONTAGION}

In the previous sections we introduced a minimal model based on a computationally highly efficient cellular automaton capable of semiquantitatively reproducing important experimental data for evacuations through a narrow exit and we showed that its results obeyed the micro-macro relation. However, so far, the pedestrian behaviors have been kept fixed, with no possibility of change due to social contagion. This deficiency is remedied in this section and we demonstrate that the presence of contagion can lead to the violation of the micro-macro relation.

In realistic conditions, the propensities to cooperate $\Pi_{i}(t)$ will vary with time, in particular under the negative influence of the aggressive acts witnessed in one's vicinity: Having a neighbor of ours choose a competitive strategy reduces our propensity to cooperation in the future; the reverse effect, that is, the positive influence of cooperative acts, is deemed weaker and is disregarded here. It is worth remarking that the existence of such contagion is endorsed by many social psychology theories, even when they claim that cooperativeness often prevails. For instance, the experimental results of Kugihara [42] supported the social identity model according to which (even in evacuation conditions) people do not break free of social constraints but conform to the salient local norms; but, more precisely, they "showed that what directly affects norm formation is the density of stimulus, that is, the amount of aggression received from others and of others' escape activity divided by group size."

Contagion occurs only among neighbors. In the model, it will be implemented by means of an equation of the form

$$
\tau_{\text {rel }} \frac{\mathcal{D} \Pi_{i}}{\mathcal{D} t}(t)=-\underbrace{\left[f\left(\Pi_{i}\right)-f\left(\Pi_{i}^{(\mathrm{intr})}\right)\right]}_{\text {relaxation }}-\underbrace{J D_{i}(t)}_{\text {contagion }},
$$

where $\frac{\mathcal{D}}{\mathcal{D} t}$ denotes a discrete time derivative (to be specified later), $\tau_{\text {rel }}$ sets the duration of the memory, $J$ is the contagion strength, $D_{i}(t) \leqslant 4$ is the number of neighbors of $i$ who chose a competitive strategy at time step $t$, and the function $f$ controls the return force of the propensity to collaborate $\Pi_{i}(t)$ to its intrinsic value $\Pi_{i}^{\text {(intr) }}$. To make the boundaries at $\Pi=0$ or 1 strongly repulsive and introduce nonlinearities in the equation, we set

$$
f(\Pi) \equiv \tan \left[\pi\left(\Pi-\frac{1}{2}\right)\right]
$$

We should mention a technical aspect associated with the discrete time derivative in Eq. (9): Defining it as a finite difference would require an excessively fine time discretization to keep $\Pi_{i}$ between 0 and 1 . Consequently, we changed variables to $\psi_{i}=f\left(\Pi_{i}\right)$, wrote $\frac{\mathcal{D} \Pi_{i}}{\mathcal{D} t}(t)=\frac{1}{f^{\prime}\left(\Pi_{i}\right)} \frac{d \psi_{i}}{d t}$, and set an arbitrary upper bound on the derivative $f^{\prime}$ to impede too sudden variations of $\psi_{i} ; \Pi_{i}(t+1)$ is then calculated as $f^{-1}\left[\psi_{i}(t+1)\right]$. This scheme allowed us to use the same discretization time step $\delta t=1$ for the behaviors and for the motion. 
Clearly, the foregoing choices are arbitrary to a large extent and we do not claim that Eq. (9) precisely describes a real contagion process; yet we will see that it yields an interesting phenomenology with plausible practical relevance. To better understand the implications of Eq. (9), consider the case in which one agent $j$ is suddenly struck with panic, so that $\Pi_{j}(t)$ reaches a value $c \ll 1$. Then, if the crowd is highly susceptible, with $J \rightarrow \infty$, the nervous or impatient behavior of this agent will contaminate others, so that (in this limit of strong contagion) the whole crowd may turn nervous.

\section{A. Analytical approach}

We now turn to a more quantitative analysis of the contagion dynamics. The analytical study is premised on a quasiequilibrium assumption: The evacuation is considered slow enough for the psychological propensities to reach their equilibrium values before any significant change in the geometric configuration of the crowd. A similar assumption was used in Ref. [13]. Under this hypothesis, in the stationary state, we can average Eq. (9) over a reasonably large number of time steps, viz.,

$$
\tau_{\text {rel }} \frac{\overline{\mathcal{D} \Pi_{i}}}{\mathcal{D} t}(t)=-\left[\overline{f\left(\Pi_{i}(t)\right)}-f\left(\Pi_{i}^{(\mathrm{intr})}\right)\right]-J \overline{D_{i}(t)},
$$

where the overbars denote time-averaged quantities.

Remarking that $D_{i}(t)=\sum_{j \in \Lambda_{i}} d_{j}(t)$, where $d_{j}(t)=1$ if agent $j$ behaved competitively at time step $t$ and 0 otherwise and $\Lambda_{i}$ denotes the first neighbors of $i$, we see that $\overline{D_{i}(t)}=$ $\sum_{j \in \Lambda_{i}}\left[1-\overline{\Pi_{j}(t)}\right]$.

Rewriting with a potential. To leading order in $\left[\Pi_{i}(t)-\right.$ $\left.\overline{\Pi_{i}(t)}\right], \overline{f\left[\Pi_{i}(t)\right]} \approx f\left[\overline{\Pi_{i}(t)}\right]$ and Eq. (11) can then be recast into

$$
\tau_{\text {rel }} \frac{\overline{\mathcal{D} \Pi_{i}}}{\mathcal{D} t}(t)=-\frac{d V_{i}}{d \overline{\Pi_{i}}}\left(\overline{\Pi_{i}}\right)-\sum_{j \in \Lambda_{i}} \frac{d V^{(2)}}{d \overline{\Pi_{i}}}\left(\overline{\Pi_{i}}, \overline{\Pi_{j}}\right),
$$

where we have introduced the potentials

$$
\begin{gathered}
V_{i}(\Pi) \equiv F(\Pi)-\Pi f\left(\Pi_{i}^{(\mathrm{intr})}\right)+z_{i} J\left(\Pi-\frac{1}{2} \Pi^{2}\right), \\
V^{(2)}\left(\Pi_{i}, \Pi_{j}\right) \equiv \frac{J}{2}\left(\Pi_{i}-\Pi_{j}\right)^{2} .
\end{gathered}
$$

Here $F$ is a primitive of $f$, e.g., $F(\Pi) \equiv-\frac{1}{\pi} \ln \mid \cos [\pi(\Pi-$ $\left.\left.\frac{1}{2}\right)\right] \mid$, and $z_{i}$ is the number of first neighbors of agent $i$. The pair potential $V^{(2)}$ is symmetric and is interpreted as an interfacial cost. Note that the foregoing reasoning is independent of the function $f(\Pi)$; accordingly, it can be used to predict the outcome of simulations based on other functional choices.

The total energy of the system is then

$$
\mathcal{V}\left(\left\{\overline{\Pi_{i}}\right\}\right)=\sum_{i} V_{i}\left(\overline{\Pi_{i}}\right)+\frac{1}{2} \sum_{(i, j) \mid j \in \Lambda_{i}} V^{(2)}\left(\overline{\Pi_{i}}, \overline{\Pi_{j}}\right) .
$$

If one neglects heterogeneities in the intrinsic distributions $\left(\Pi_{i}^{\text {(intr) }} \rightarrow \Pi^{\text {(intr) }}\right)$ and in the configuration $\left(z_{i} \rightarrow z\right), V_{i}$ becomes independent of $i\left(V_{i} \rightarrow V\right)$ and the ground state $\left\{\Pi_{j}\right\}$ for the energy is obtained for the homogeneous system at $\Pi_{j}=$

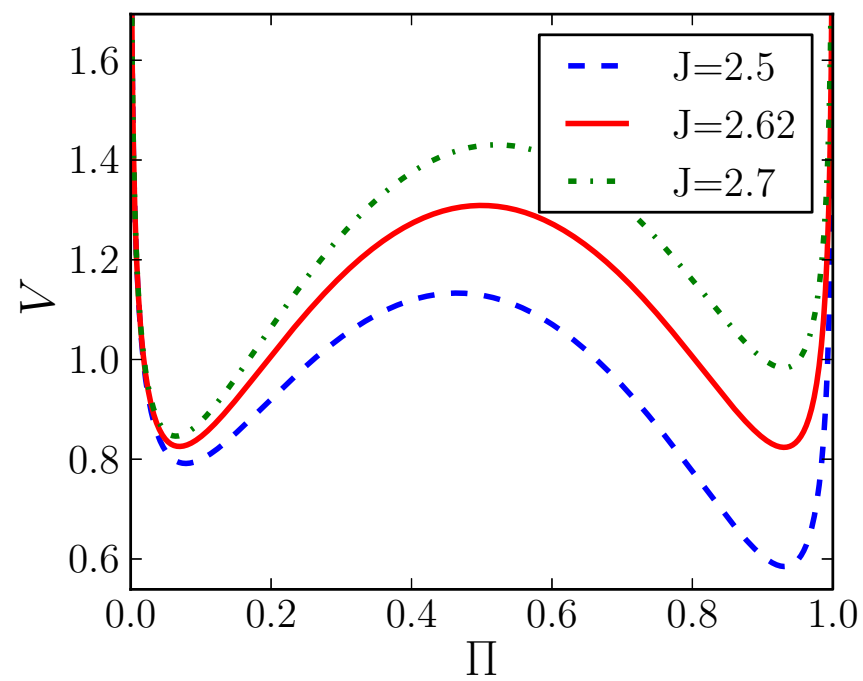

FIG. 5. Potential $V$ for $\Pi^{(\text {intr })}=0.93, z=4$, and different coupling parameters $J$, as indicated in the legend.

$\Pi$, where $\Pi$ minimizes $V$, viz., $\frac{d V}{d \Pi}=0$. Interestingly, for some values of the coupling parameter $J$ and $\Pi^{(\text {intr) }}$, the potential $V$ displays bistability (see Fig. 5), with two distinct minima at $\Pi_{\downarrow}$ and $\Pi_{\uparrow}$ such that, by definition, $V\left(\Pi_{\downarrow}\right)<V\left(\Pi_{\uparrow}\right)$. For $z=4$ and $\Pi^{\text {(intr) }}=0.94$, the minima are approximately of equal depths when $J \simeq 2.62$.

Since, at not too high temperature, all $\Pi_{j}$ will dwell close to the bottom of an energy basin, it is convenient to write $\Pi_{j}=\Pi_{\downarrow}+\tilde{\Pi}$ as $(\downarrow, \tilde{\Pi})\left[\right.$ or $\Pi_{j}=\Pi_{\uparrow}+\tilde{\Pi}$ as $\left.(\downarrow, \tilde{\Pi})\right]$ in the bistable state. If the thermal deviations $\tilde{\Pi}$ are overlooked, the model can be mapped directly onto a two-dimensional (2D) Ising model in an external field $h$, with the Hamiltonian

$$
\mathcal{H}=-h \sum_{i} \sigma_{i}-\frac{J_{\text {Ising }}}{2} \sum_{(i, j) \mid j \in \Lambda_{i}} \sigma_{i} \sigma_{j},
$$

and $\sigma_{i}, \sigma_{j} \in\{-1,1\}$, with

$$
\begin{aligned}
h & =\frac{V\left(\Pi_{\downarrow}\right)-V\left(\Pi_{\uparrow}\right)}{2}, \\
J_{\text {Ising }} & =\frac{J}{4}\left(\Pi_{\uparrow}-\Pi_{\downarrow}\right)^{2} .
\end{aligned}
$$

It is well known that, in the absence of an external field $h$, the 2D Ising system undergoes a phase transition from a disordered state with mixed spins to an ordered ( $\uparrow$ or $\downarrow$ ) state as the temperature declines and that the transition is associated with diverging correlation lengths. The thermodynamic transition disappears at $h \neq 0$; nevertheless, a vestige of the criticality persists at small but finite $h$.

There are no bona fide thermal fluctuations in Eq. (9), but the instantaneous discrepancies between $d_{j}(t) \in\{0,1\}$ and $1-\overline{\Pi_{j}(t)}$ introduce fluctuations $\xi(t)$ in practice, viz.,

$$
\tau_{\text {rel }} \frac{\mathcal{D} \Pi_{i}}{\mathcal{D} t}(t)=-\frac{d V_{i}}{d \overline{\Pi_{i}}}\left(\overline{\Pi_{i}}\right)-\frac{1}{2} \sum_{(i, j) \mid j \in \Lambda_{i}} \frac{d V^{(2)}}{d \overline{\Pi_{i}}}\left(\overline{\Pi_{i}}, \overline{\Pi_{j}}\right)+\xi(t) .
$$

By comparing the first and second moments of $\xi$, namely, $\langle\xi\rangle=0$ and $\left\langle\xi^{2}\right\rangle \approx 4 J^{2} \overline{\Pi_{i}}\left(1-\overline{\Pi_{i}}\right)$, with the thermal relation 

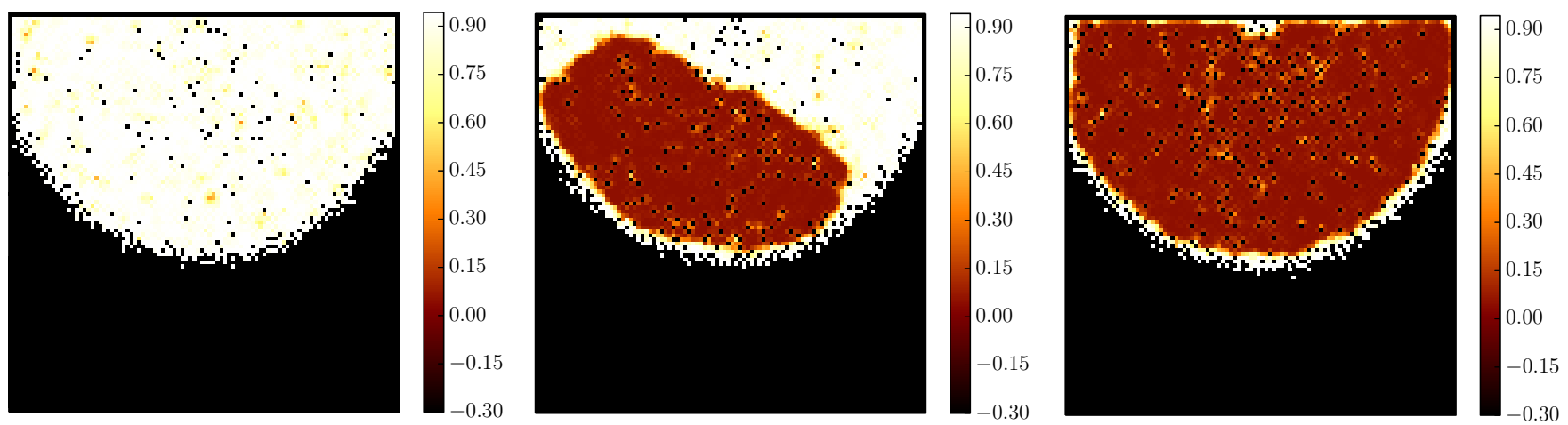

FIG. 6. Color maps of the propensities $\Pi_{j}(t)$ in the crowd at time $t=4000$ for $\Pi^{\text {(intr) }}=0.94 \pm 10^{-7}$ and $J=2.96$ (left), $J=2.9704$ (middle), and $J=2.98$ (right). Vacant sites appear in black.

$\left\langle\xi^{2}\right\rangle=2 \tau_{\text {rel }} T$, we get a lower bound for the effective temperature $T_{\mathrm{eff}}=\frac{2 J^{2}}{\tau_{\mathrm{rel}}} \overline{\Pi_{i}}\left(1-\overline{\Pi_{i}}\right)$.

\section{B. Numerical results}

Numerical simulations of the model confirm the validity of the foregoing analysis. Snapshots of the propensities of the evacuating crowd are shown in Fig. 6 as color maps, for different values of the coupling parameter $J$. Clearly, as $J$ is varied across a critical value $J^{\star}$, large domains of strongly correlated propensities are observed in the system, which is suggestive of the close presence of a critical point. Two additional spatial feature are noticeable. There is an abundance of cooperative (high- $\Pi$ ) agents in the vicinity of the exit, along with a cooperative fringe at the outer edge of the crowd, where each agent has fewer neighbors, i.e., lower coordination $(z)$ values, and thus fewer contagion possibilities.

These results hold only for a sharply peaked distribution of intrinsic propensities $\Pi^{\text {(intr) }}$; for more broadly distributed

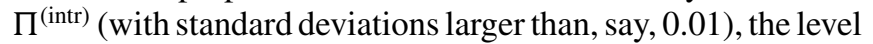
of disorder increases and the spatial correlations of the propensity become less visible at $J^{\star}$. As the size $L$ of the system decreases, the border and vacant sites become proportionally
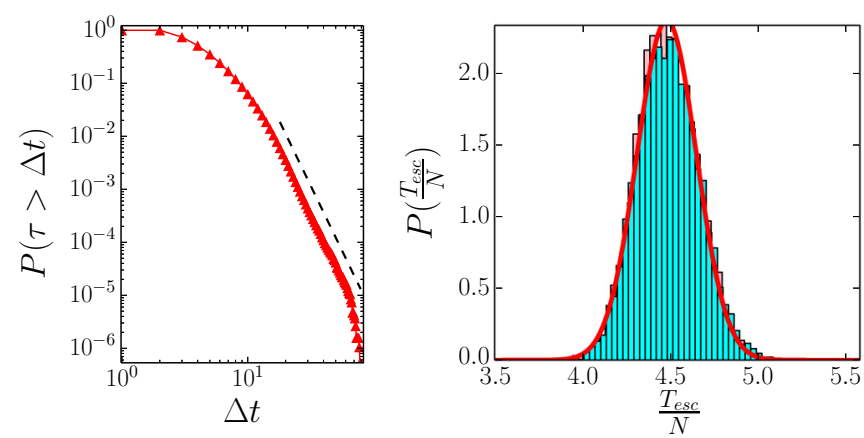

FIG. 7. Evacuations in the presence of behavioral contagion for $J=3.35$ and $\Pi^{\text {(intr) }}=0.94$ in a system of size $L=25$. Shown on the left is the survival function and on the right the normalized global distribution of evacuation times per pedestrian $\frac{T_{\text {esc }}}{N}$, with the simulations in light blue, the expectations from the micro-macro relation in hatched pink (see the text), and the Gaussian distribution of Eq. (3) as a solid red curve. more important, so the critical contagion strength $J^{\star}(L)$ at the onset of the evacuation shifts to larger values; for example, we observed that $J^{\star}(L=100) \approx 2.97, J^{\star}(L=40) \approx 3.11$, and $J^{\star}(L=25) \approx 3.33$. This notably implies that the critical value $J^{\star}$ will increase during the evacuation as fewer and fewer people are left in the room.

Let us now move on to the possible effect of the contagion rule on the evacuation.

The existence of large correlated domains in the unbounded system implies that, should the system be small enough, i.e., of order the correlation length or below, there will be occurrences where the crowd will escape cooperatively and others, where panic will spread and foster selfish behavior. We claim that, notwithstanding the arbitrariness of the chosen rules, this behavioral dichotomy is a practically relevant general effect due to social contagion. In particular, taking it into account may help settle the debate between the staunch supporters of the maintenance of social cooperation in emergency evacuations $[22,43]$ and the proponents of the emergence of selfish or aggressive behaviors ${ }^{4}$ in emergency, as often implemented in physics-based models [12,13,15,34,39].

We turn to the micro-macro relation. The microscopic distribution of time lapses $\Delta t$, averaged over many realizations, is plotted in Fig. 7 for a system close to the critical point, $J \simeq$ $J^{\star}(L)$ and $L=25$. Despite the presence of correlated domains in the simulations (not shown), the Gaussian prediction of Eq. (3) based on the micro-macro relation in the limit $N \rightarrow \infty$ seems to capture the actual distribution of global evacuation evacuation times satisfactorily. To test the agreement more thoroughly, we measured the distance between the distribution of $N_{\text {sim }}$ simulated evacuation times and the distribution of $N_{\text {sim }}$ sums of $N$ time lapses randomly drawn from the microscopic distribution. As a statistical measure of distance (or rather proximity) between finite-size distributions, we chose the $p$ value associated with either the Mann-Whitney test or the Kolmogorov-Smirnov test. Despite collecting about $N_{\text {sim }} \approx$ 5000 global evacuation times for every set of parameters

\footnotetext{
${ }^{4}$ Conversely, the effect of cohesion enhancement through identification within the group predicted by the social identity theory would be accounted for by an increase in $\Pi^{\text {(intr) }}$ and possibly by a dependence of these propensities on the neighbors.
} 

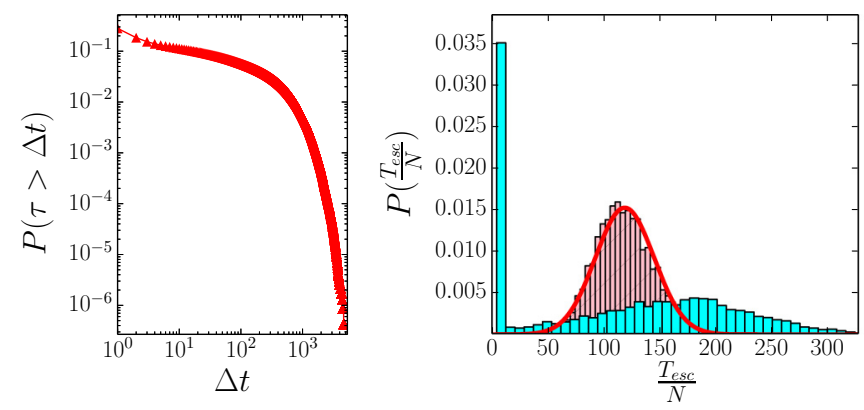

FIG. 8. Evacuations in the presence of behavioral contagion for $J=453$ and $\Pi^{\text {(intr) }}=0.99905$ in a system of size $L=30$. The rest is the same as in Fig. 7.

and averaging over many random distributions, we did not measure any $p$ value sufficiently small to ascertain a significant difference between the actual distribution and the random one, nor did we observe variations of the $p$ value with the contagion strength $J$ that were substantially above the statistical noise. Therefore, if the micro-macro relation is violated, the violation is at most tenuous. This could be due to the finite lifetime of the domains of correlated propensities or to the aforementioned persistence of cooperative behaviors near the exit (compared to the bulk), where time lapses are computed.

Thus, deviations from the micro-macro relation in the bistable system considered above are, at best, moderate. Let us now consider another mechanism that may induce deviations, namely, the scenario (mentioned at the end of Sec. II) of an initially cooperative crowd that is highly susceptible to panic, i.e., with very large $J$. In this case, the crowd is initially trapped in a metastable high- $\Pi$ state and the question is whether, in a given realization, nervous or impatient behaviors will have time to nucleate and push the crowd into the stable low- $\Pi$ state, thus delaying the evacuation (all the more so as these behaviors have nucleated early), or not. Figure 8 presents the microscopic and macroscopic distributions associated with this scenario. Once again, the macroscopic distribution is compared with a random distribution inferred from the microscopic statistics. Here the discrepancy is blatant, which reflects the dramatic failure of the micro-macro relation. In particular, the inferred distribution captures neither the frequency of fast (cooperative) evacuations nor the occurrence of sluggish evacuations where competitive behaviors pervade the crowd.

\section{DISCUSSION AND POTENTIAL RELEVANCE FOR SAFETY SCIENCE}

It is a crucial requirement in any safety protocol that buildings should provide adequate means of egress in the event of an evacuation, by preserving unlocked, unobstructed, and clearly marked emergency exits. There is a plethora of historical examples where an insufficient outflow capacity was reported as a decisive factor leading to a crowd disaster. Indeed, in their haste to exit, pedestrians may tend to push their neighbors, which can result in the buildup of pressure at the exit and its congestion. Recent experimental works have raised the hope of a quantitative characterization of the outflow capacity of a given exit, based on the distribution of time lapses between successive egresses and depending on the eagerness to egress.

In the present work we have developed a minimalistic cellular automaton model able to reproduce semiquantitatively the major features observed in (controlled) evacuation experiments. In particular, for competitive egresses we obtained a distribution exhibiting a large tail that is well described by a power law. For sure, our model is but a minimal abstraction of the real problem; still, it is noteworthy that our approach is in line with more detailed previous studies that endeavored to go beyond the reliance of the social force paradigm on binary interactions between pedestrians. In [44] the authors considered that, instead of being repelled by their neighbors, the individuals look for a free path through the crowd. In this sense, our cellular automaton is based on rather similar considerations.

Among other features, we focused on the effect of social contagion. As expected, in its absence, the (practically relevant) macroscopic distribution of global evacuation times is reliably inferred from the microscopic statistics of time lapses. On the contrary, should there be a possibility of behavioral contagion, this micro-macro relation may be violated due to the induced correlations in the behaviors of the pedestrians within each evacuation. While its origin can be attributed to complex causes and its study lies in the realms of social psychology, we have abstracted and illustrated this point by implementing simple contagion dynamics, which turned out to be quantitatively connected to the 2D Ising model: In some regions of parameter space, the crowd displays bistability, with a cooperative state and an impatient one. However, large violations of the micro-macro relation were in fact only observed at very large contagion strengths, when the crowd initially resided in a metastable cooperative state.

This observation is conceptually interesting in that it shows that, even in strictly identical conditions, the durations of evacuation may vary much more than suggested by naive reasoning (the micro-macro relation), although numerically this happened only in a select region of parameter space; the higher variability was then due to contagion-induced correlations.

In reality, it is highly unlikely that two evacuations take place in strictly identical conditions: Crowds will differ in their composition and their psychological states will be strongly affected by previous circumstances, not to mention the singularity of each evacuation and the various possible emergency stimuli. Within the simple framework of our model, these differences might be accounted for by variations of both the distribution of intrinsic probabilities $\Pi^{\text {(intr) }}$ and the contagion strength $J$ with the occurrence. In any event, it is natural to expect that this enhanced variability between realizations will result in more systematic deviations from the micro-macro relation than in our study.

Given this failure of the micro-macro relation, should experts in safety science dedicate any attention whatsoever to the microscopic distribution of time lapses (which, unlike the global one, can potentially be compiled)? As a matter of fact, we believe that this approach still represents a step forward with respect to the traditional use of a single flow rate value (e.g., an exit capacity of 82 persons per meter width per minute, according to the British Guide to Safety at Sports Grounds [45]), in that it accounts for statistical fluctuations (but not for 
context-related variations). In this spirit, one may envision a conservative approach to the assessment of the exit capacities. Regardless of the geometry, it would consist in extracting the microscopic distribution from the slowest evacuations of a densely crowded room, from a batch of real video-recorded evacuations, and deriving the global distribution from the micro-macro relation, as a function of the attendance. Data from evacuation drills might also be considered, but this demands to find a trade-off in the dilemma between prescribing realistically competitive behaviors to the participants and ensuring a safe drill. Alternatively, the avoidance of worst-case scenarios in real evacuations could be left to the responsibility of other mitigating strategies (i.e., not related to the geometry); then, under the assumption that the evacuating crowd does not yield to panic, typical egresses could be used to compile the microscopic statistics and derive the exit capacity. Finally, it is worth recalling that the possibilities of delay contemplated here arise because of congestion at the exit and are naturally less pronounced with wider doors or a scarcer crowd.

On a more general note, our work is yet another example of the potential of statistical physics models to provide a phenomenological account of society-related topics [12,46,47]. Along this line, we remark that, both in the (statistical) physics of complex systems and in the social psychology of crowds $[48,49]$, many subtleties of the individual entity are left behind when the entity forms part of an assembly and the collective response may be more polarized than that of the isolated entities.

\section{ACKNOWLEDGMENTS}

This work received financial support from CONICET (Grant No. PIP 112-201101-0310) and Universidad Nacional de Cuyo (Grant No. 06/C304).

\section{APPENDIX A: LIMIT DISTRIBUTIONS FOR POWER-LAW EXPONENTS $\alpha \leqslant 3$}

Consistently with empirical measurements of the distribution $p(\Delta t)$ of time lapses $\Delta t$ between successive egresses, in the main text we focused on distributions featuring a power-law tail $p(\Delta t) \sim \Delta t^{-\alpha}$ with an exponent $\alpha>3$. In that case, the sum of time lapses converges to a Gaussian distribution, as stated by the central limit theorem. However, what happens if $\alpha \leqslant 3$ ? For $2<\alpha \leqslant 3$, the mean value of the distribution is still well defined, but the standard deviation diverges and a Lévy distribution is expected for the sum of the time lapses.

Indeed, according to the developments in [50,51] and restricting our attention to the case $2<\alpha \leqslant 3$, we find the following result. Let $p(\Delta t)$ behave as $p(\Delta t)=\lambda \Delta t^{-\alpha}$ for large $\Delta t$ and let $\mu$ be its mean value. Then the distribution of $T_{\text {esc }}=\sum_{i=1}^{N} \Delta t_{i}$ for large $N$ converges to the Lévy density function

$$
f\left(T_{\mathrm{esc}} \mid \tilde{\alpha}, 1, \gamma_{N}, \delta_{N}, 0\right),
$$

with the notation and parameter prescriptions of Ref. [50], where $\tilde{\alpha} \equiv \alpha-1(1<\tilde{\alpha} \leqslant 2), \delta_{N} \equiv N \mu+\gamma_{N} \tan \frac{\pi \tilde{\alpha}}{2}$, and

$$
\gamma_{N} \equiv\left(\frac{\pi \lambda N}{2 \tilde{\alpha} \sin \left(\frac{\pi \tilde{\alpha}}{2}\right) \Gamma(\tilde{\alpha})}\right)^{1 / \tilde{\alpha}}
$$

Recall that this result is conditioned by the validity of the micro-macro connection, which may fail in the presence of contagion, as discussed in the main text.

\section{APPENDIX B: APPROXIMATE ANALYTICAL ESTIMATION OF THE MICROSCOPIC DISTRIBUTION}

In the main text we presented simulations of the evacuation of a strongly competitive crowd through a narrow door of unit width. Numerically, the distribution $p(\Delta t)$ of time lapses $\Delta t$ was found to be well fitted by a power law of exponent $\alpha \approx 3.7$ at large $\Delta t$. Here we aspire to support these numerical results with approximate analytical calculations.

First, the rules of the model imply that the largest time lapses are obtained when three very impatient agents $i, j$, and $k\left(\Pi_{i}, \Pi_{j}, \Pi_{k} \ll 1\right)$ compete for the site just in front of the exit. In the worst cases, the sites behind them are also occupied, which deprives them of the option of stepping backward. Most probably, the conflict will be resolved only when two of these agents accept to stay on their current sites, while the third one attempts to move forward to the desired site.

For $\Pi_{i} \ll 1$, considering the probabilities of site selection of Eq. (5) and the attractiveness defined in Eqs. (6) and (7) with $k=0.5$ and $\eta=1$, the most probable option that leads agent $i$ to stay on site is not to choose a cooperative strategy, but rather to adopt a competitive behavior (with probability $1-\Pi_{i}$ ) and then select the current site (with probability $p_{i}^{s}$ of order $e^{-1} / \eta e^{k \ln \Pi_{i} / \eta} \sim \Pi_{i}^{k / \eta}=\sqrt{\Pi_{i}}$ ). To leading order in the propensities and up to numerical prefactors, the probability $P(\Delta t=n)$ of observing a time lapse of duration $n \gg 1$ is given by the probability of a succession of $n-1$ conflicts finally resolved by two agents choosing to stay on site, viz.,

$$
\int d \Pi_{i} D\left(\Pi_{i}\right) \int d \Pi_{j} D\left(\Pi_{j}\right) \int d \Pi_{k} D\left(\Pi_{k}\right)\left(1-s^{(2)}\right)^{n-1} s^{(2)},
$$

where $D\left(\Pi_{\gamma}\right), \gamma \in\{i, j, k\}$, is the distribution of propensities and $s^{(2)}$ is shorthand for $\left(p_{i}^{s} p_{j}^{s}+p_{j}^{s} p_{k}^{s}+p_{i}^{s} p_{k}^{s}\right)$. For the strongly competitive crowd, it is fair to approximate the truncated Gaussian distribution $D\left(\Pi_{\gamma}\right)$ by a constant for $\Pi_{\gamma}<\epsilon$, where $\epsilon$ is a tiny constant. Changing variables $\Pi_{\gamma}$ to $p_{\gamma}^{s} \equiv \sqrt{\Pi_{\gamma}}$, we get

$$
\begin{aligned}
P(\Delta t=n) \sim & \int_{0}^{\epsilon} d p_{i}^{s} p_{i}^{s} \int_{0}^{\epsilon} d p_{j}^{s} p_{j}^{s} \\
& \times \int_{0}^{\epsilon} d p_{k}^{s} p_{k}^{s}\left(1-s^{(2)}\right)^{n-1} s^{(2)} .
\end{aligned}
$$

The triple integral can be split into a region $p_{k}^{s}<p_{j}^{s}<p_{i}^{s}$ and five other symmetric regions. In the first region, we make the approximation $s^{(2)} \approx p_{i}^{s} p_{j}^{s}$ so that, up to numerical factors,

$$
\begin{aligned}
P(\Delta t=n) \sim & \int_{0}^{\epsilon} d p_{i}^{s} \int_{0}^{p_{i}^{s}} d p_{j}^{s} \int_{0}^{p_{j}^{s}} d p_{k}^{s}\left(1-p_{i}^{s} p_{j}^{s}\right)^{n-1} \\
& \times\left(p_{i}^{s}\right)^{2}\left(p_{j}^{s}\right)^{2} p_{k}^{s} .
\end{aligned}
$$

Finally, the integrand reaches its maximum at $p_{i}^{s} \approx p_{j}^{s} \approx p_{k}^{s} \sim$ $n^{-1 / 2}$ and rapidly decays for larger $p_{i}^{s}$ so that, discarding the part $p_{i}^{s}>n^{-1 / 2}$ and approximating the integrand by its 
maximum, we arrive at

$$
P(\Delta t=n) \sim n^{-4},
$$

that is to say, the distribution of time lapses decays as a power law with exponent $\alpha=4$. Considering the many inaccuracies involved in the foregoing calculation, we deem the agreement between the analytical result and the numerical one $(\alpha \simeq 3.7)$ quite satisfactory.

\section{APPENDIX C: POWER-LAW FITS}

As stressed by Clauset et al. [37], some care needs to be taken when fitting empirical data with power laws, insofar as simple graphical representations of the empirical distribution function may be misleading. Indeed, before implementing the cellular automaton described in the main text, we worked with a slightly different version (which, in particular, did not feature disorder in the intrinsic probabilities $\left.\Pi^{(\text {intr) }}\right)$. A logarithmic plot of the distribution of time lapses obtained with that model [see Fig. 9(a)] made us think that the data were well described by a power-law tail. However, after plotting the complementary cumulated distribution (i.e., the survival function), we realized that the data were in fact better described by an exponential distribution [compare Fig. 9(b) with the red dotted lines in Fig. 1].
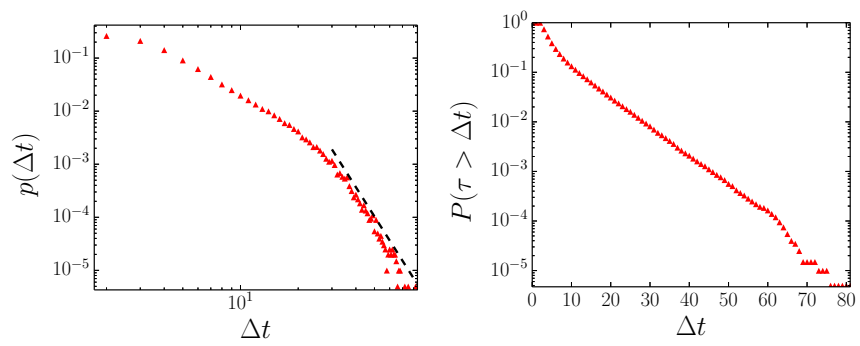

FIG. 9. Graphical representations of the distribution function obtained with the contagion-free model for a strongly competitive crowd, where the heterogeneous distribution of $\Pi^{\text {intr }}$ is replaced by a Dirac peak. Shown on the left is the probability distribution function in a logarithmic plot and on the right the survival function in a semilogarithmic plot.

This experience cast some doubt in our minds about the observations of power-law distributions of time lapses with cellular automata reported in the literature [52,53]. By plotting the survival functions of the data shown in these works and applying the methods of Refs. [36,37] to compute the likelihood to be a power law, we found that the data of Ref. [53] and, to a lesser extent, Ref. [52] are at least as compatible with an exponential tail as they are with a power-law one.
[1] K. Takimoto and T. Nagatani, Physica A 320, 611 (2003).

[2] M. Casanova, Resúmen Histórico del Gran Incendio de la Compañía (Librería Española de Nicasio Ezquerra, Valparaíso, 1863).

[3] X. Pan, C. S. Han, K. Dauber, and K. H. Law, Automat. Construct. 15, 448 (2006).

[4] A. P. Hatch, Tinder Box: The Iroquois Theatre Disaster 1903 (Chicago Review, Chicago, 2003).

[5] C. E. Nicholson and B. Roebuck, Safety Sci. 18, 249 (1995).

[6] J. Dickie, Safety Sci. 18, 309 (1995).

[7] D. Helbing and P. Mukerji, EPJ Data Sci. 1, 1 (2012).

[8] Tragedia en un boliche de once, Diario Clarín (31 December 2004).

[9] Bucharest nightclub fire: Death toll reaches 45 as drummer of band dies, The Guardian (8 November 2015), https://www. theguardian.com/world/2015/nov/08/bucharest-nightclubblaze-death-toll-reaches-45-drummer-metal-band-dies.

[10] I. Zuriguel, D. R. Parisi, R. C. Hidalgo, C. Lozano, A. Janda, P. A. Gago, J. P. Peralta, L. M. Ferrer, L. A. Pugnaloni, E. Clément et al., Sci. Rep. 4, 7324 (2014).

[11] J. M. Pastor, A. Garcimartín, P. A. Gago, J. P. Peralta, C. MartínGómez, L. M. Ferrer, D. Maza, D. R. Parisi, L. A. Pugnaloni, and I. Zuriguel, Phys. Rev. E 92, 062817 (2015).

[12] X. Zheng and Y. Cheng, Physica A 390, 1042 (2011).

[13] S. Heliövaara, H. Ehtamo, D. Helbing, and T. Korhonen, Phys. Rev. E 87, 012802 (2013).

[14] S. Bouzat and M. N. Kuperman, Phys. Rev. E 89, 032806 (2014).

[15] A. von Schantz and H. Ehtamo, Phys. Rev. E 92, 052805 (2015).

[16] A. H. Buchanan, Fire Engineering Design Guide (University of Canterbury, Christchurch, 2001).
[17] D. A. Purser and M. Bensilum, Safety Sci. 38, 157 (2001).

[18] N. R. Johnson, Soc. Probl. 34, 362 (1987).

[19] A. R. Mawson, Psychiatry 68, 95 (2005).

[20] B. E. Aguirre, Psychiatry 68, 121 (2005).

[21] J. Drury, C. Cocking, S. Reicher, A. Burton, D. Schofield, A. Hardwick, D. Graham, and P. Langston, Behav. Res. Methods 41, 957 (2009).

[22] J. Drury, C. Cocking, and S. Reicher, Brit. J. Soc. Psychol. 48, 487 (2009).

[23] A. Garcimartín, I. Zuriguel, J. Pastor, C. Martín-Gómez, and D. Parisi, Transp. Res. Proc. 2, 760 (2014).

[24] Video available at https://www.youtube.com/watch?v=u2LO mnldQkk

[25] S. Marshall, Men Against Fire (University of Oklahoma Press, Norman, 1947).

[26] S. Chen, F. Alonso-Marroquin, J. Busch, R. Cruz-Hidalgo, C. Sathianandan, Á. Ramírez-Gómez, and P. Mora, in Powders and Grains 2013: Proceedings of the Seventh International Conference on Micromechanics of Granular Media, edited by A. Yu, K. Dong, R. Yang, and S. Luding, AIP Conf. Proc. No. 1542 (AIP, New York, 2013), p. 157.

[27] C. Burstedde, K. Klauck, A. Schadschneider, and J. Zittartz, Physica A 295, 507 (2001).

[28] M. Moussaïd, N. Perozo, S. Garnier, D. Helbing, and G. Theraulaz, PLoS ONE 5, e10047 (2010).

[29] A. Kirchner, K. Nishinari, and A. Schadschneider, Phys. Rev. E 67, 056122 (2003).

[30] K. To, P.-Y. Lai, and H. K. Pak, Phys. Rev. Lett. 86, 71 (2001).

[31] A. Garcimartín, I. Zuriguel, L. A. Pugnaloni, and A. Janda, Phys. Rev. E 82, 031306 (2010). 
[32] C. Lozano, A. Janda, A. Garcimartín, D. Maza, and I. Zuriguel, Phys. Rev. E 86, 031306 (2012).

[33] A. Garcimartín, J. M. Pastor, L. M. Ferrer, J. J. Ramos, C. Martín-Gómez, and I. Zuriguel, Phys. Rev. E 91, 022808 (2015).

[34] D. Helbing, I. Farkas, and T. Vicsek, Nature (London) 407, 487 (2000).

[35] D. Parisi and C. Dorso, Pedestrian and Evacuation Dynamics (Springer, Berlin, 2007), pp. 341-346.

[36] J. Alstott, E. Bullmore, and D. Plenz, PLoS ONE 9, e85777 (2014).

[37] A. Clauset, C. R. Shalizi, and M. E. Newman, SIAM Rev. 51, 661 (2009).

[38] Y. Lizhong, F. Weifeng, and F. Weicheng, J. Fire Sci. 21, 227 (2003).

[39] X. Song, L. Ma, Y. Ma, C. Yang, and H. Ji, Physica A 447, 455 (2016).

[40] G. J. Perez, G. Tapang, M. Lim, and C. Saloma, Physica A 312, 609 (2002).

[41] A. Seyfried, O. Passon, B. Steffen, M. Boltes, T. Rupprecht, and W. Klingsch, Transport. Sci. 43, 395 (2009).

[42] N. Kugihara, Brit. J. Soc. Psychol. 40, 575 (2001).

[43] J. Drury, Collective Resilience in Mass Emergencies and Disasters (Psychology Press, Hove, 2012).
[44] M. Moussaï, D. Helbing, and G. Theraulaz, Proc. Natl. Acad. Sci. USA 108, 6884 (2011).

[45] Football League Authority, Guide to Safety at Sports Grounds, 5 th ed. (Department for Culture, Media and Sports, Norwich, 2008).

[46] Y. Xue, Y.-f. Wei, H.-h. Tian, and L.-j. Liang, in Pedestrian and Evacuation Dynamics 2008, edited by W. W. F. Klingsch, C. Rogsch, A. Schadschneider, and M. Schreckenberg (Springer, Berlin, 2010), pp. 371-379.

[47] J.-P. Bouchaud, J. Stat. Phys. 151, 567 (2013).

[48] G. Le Bon, Psychologie des Foules (Sparkling Books, Southampton, 1895).

[49] N. R. Johnson, J. G. Stemler, and D. Hunter, Sociometry 40, 183 (1977).

[50] J. Nolan, Stable Distributions: Models for Heavy-Tailed Data (Springer, New York, 2016).

[51] B. Gnedenko and A. Kolmogorov, Limit Distributions for Sums of Independent Random Variables (Addison-Wesley, Reading, 1968).

[52] W. Song, Y. Yu, W. Fan, and H. Zhang, Sci. China Ser. E: Eng. Mater. Sci. 48, 403 (2005).

[53] S. Wei-Guo, Y. Yan-Fei, W. Bing-Hong, and F. Wei-Cheng, Physica A 371, 658 (2006). 